\title{
Fluctuations and jump dynamics in atomic friction experiments
}

\author{
S. Maier,* Yi Sang, T. Filleter, M. Grant, and R. Bennewitz ${ }^{\dagger}$ \\ Department of Physics, McGill University, Montreal, Quebec, Canada \\ E. Gnecco and E. Meyer \\ Department of Physics and Astronomy, University of Basel, Switzerland \\ (Received 26 August 2005; revised manuscript received 13 October 2005; published 15 December 2005)

\begin{abstract}
Atomic stick-slip processes have been studied in detail by means of friction force microscopy with high spatial and temporal resolution. The influence of the tip-sample contact on the thermal fluctuations of the force sensor and on the dynamics of the stick-slip process are characterized. Results are compared with simulations based on an extended Tomlinson model including thermal fluctuations. A correlation between the duration of the atomic slip event and the atomic structure of the contact is established.
\end{abstract}

DOI: 10.1103/PhysRevB.72.245418

PACS number(s): 68.35.Af, 07.79.Sp, 46.55.+d

\section{INTRODUCTION}

Friction force microscopy studies tribological properties of nanometer-scale, single asperity contacts. By reducing the complexity of tribological problems arising for rough surfaces in contact to one single contact, the method contributes to the understanding of basic mechanisms of adhesion, friction, and mechanical energy dissipation. When the sharp tip of the force microscope slides over a flat surface, lateral forces as small as some tenths of nanonewtons can be measured which reflect the interaction of a very small number of atoms.

Already Mate et al. described in their report on friction force microscopy a stick-slip movement of the tip with the atomic periodicity of the samples surface structure. ${ }^{1}$ This atomic stick-slip process has attracted a lot of attention since it can be seen as an elementary mechanism of friction. The tip is locked to one atomic position at the surface until the increasing lateral force is strong enough to initiate a sudden jump to the next position. In a recent paper, we have demonstrated how the occurrence of the stick-slip behavior is one-to-one related to energy loss in the sliding process. ${ }^{2}$ When reducing the normal load on the contact, the tip starts to slide continuously over the surface potential and friction is reduced essentially to zero. Only at increased normal load the stick-slip instabilities arise and cause energy dissipation. An alternative pathway to avoid stick-slip has been realized by Dienwiebel et al., who found zero friction when they rotated the contact such that the atomic structures of surface and sliding tip became incommensurable. ${ }^{3}$ Other recent results include the understanding of the role of thermal activation for the velocity dependence of atomic friction. ${ }^{4-7}$ The release of the slip instability due to thermal fluctuations reduces friction at elevated temperatures, and at slow sliding velocities.

All these results indicate the key role of the slip instability for the dissipation of mechanical energy into heat. In this paper we describe experiments and simulations which address in detail the dynamics of the atomic-scale slip and role of thermal fluctuations of the force sensor for the process. The relatively simple structure of the cantilever-beam type force sensor serves as a model system for the interplay be- tween microscopic contact and macroscopic body which is part of the general problem of understanding friction. After introducing our experimental methods we present results for the fluctuations of the force signals and the dynamic of the stick-slip process. We then describe results of a simulation for a one-dimensional model of the process and finally compare and discuss the results.

\section{EXPERIMENTAL SETUP}

The friction force acting on a nanometer-sized tip was measured by means of a home-built atomic force microscope in ultrahigh vacuum (UHV) at room temperature. The cantilever deflection was detected using the beam-deflection scheme and position-sensitive photodiode. A fast preamplifier was integrated into ultrahigh vacuum in order to keep low-voltage signal lines from the photodiode very short. This effort provides a high bandwidth of $3 \mathrm{MHz}$ for force measurements, which is sufficient to detect the torsional resonance frequency of the cantilever. We recorded the forces while scanning the tip over the surface employing a commercial scan controller (SCALA, OMICRON nanotechnology). Selected time series of the lateral force were simultaneously recorded with a sampling rate of up to $20 \mathrm{MHz}$ using a digital oscilloscope card (National Instruments).

Silicon cantilevers bearing sharp tips with a spring constant $k_{N}=0.09 \mathrm{~N} / \mathrm{m}$ for normal bending and $k_{T}=62 \mathrm{~N} / \mathrm{m}$ for torsion were used for atomic friction experiments. These spring constants were calculated for the clamped beam geometry using optical microscopy data for the length and the width of the beam and the frequency of the first normal bending mode to determine its thickness. The sensitivity of the photodiode was determined by recording force-distance curves on a hard surface. All normal forces are given with respect to the unbent cantilever, negative normal forces refer to a up bending of the cantilever that partially compensates adhesion forces. The experiments were performed on a $\mathrm{KBr}(100)$ single crystal, which was cleaved in air, transferred immediately to UHV, and annealed at $120^{\circ} \mathrm{C}$ for 30 min in order to remove contaminants.

For load dependence studies we also used an alternative set of silicon cantilevers bearing a spherical tip (Nanosen- 

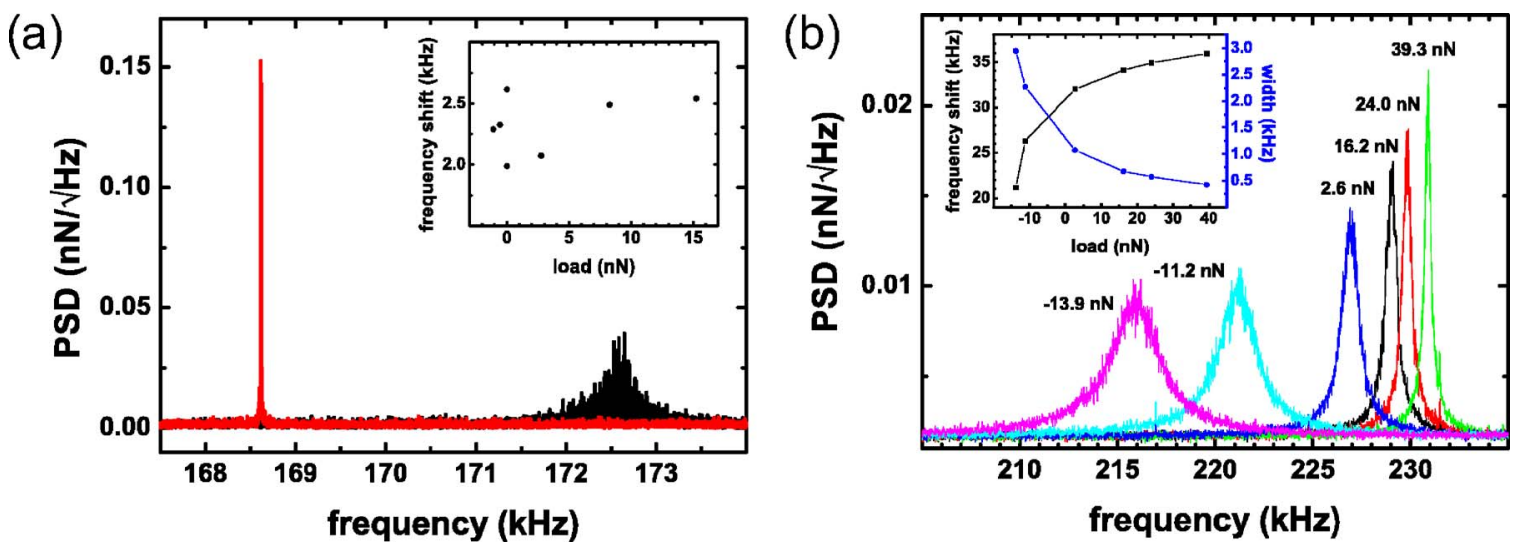

FIG. 1. (Color online) (a) Power spectral density (PSD) of thermal fluctuations in the lateral force signal showing peaks corresponding to the first torsional resonance for the free cantilever (narrow peak) and for the cantilever with a sharp tip in contact with an applied normal force of $-0.1 \mathrm{nN}$ (wider peak). The inset shows the frequency shift between free cantilever and cantilever in contact for different applied loads. Note that no significant load dependence is found. (b) Power spectral density of the lateral force signal from a cantilever bearing a micrometer-scale spherical tip in contact with an $\mathrm{Al}_{2} \mathrm{O}_{3}$ surface for different loads. The center frequency increases monotonously with higher loads, while the width of the peaks decreases as indicated in the inset. Note that the total area of the peaks is not constant. The torsional resonance frequency of the free cantilever was $195.0 \mathrm{kHz}$.

sors). The radius of curvature of the sphere was determined by scanning electron microscopy to be $0.9 \mu \mathrm{m}$. The spring constant of the cantilevers with spherical tip were $k_{N}$ $=0.11 \mathrm{~N} / \mathrm{m}$ for normal bending and $k_{T}=82 \mathrm{~N} / \mathrm{m}$ for torsion. Load dependence experiments with this tip were performed on a $\mathrm{Al}_{2} \mathrm{O}_{3}(0001)$ single crystal which guaranteed negligible deformation of the sample. The $\mathrm{Al}_{2} \mathrm{O}_{3}(0001)$ sample was heated in air at $1400{ }^{\circ} \mathrm{C}$ for four hours and in an UHV at $120^{\circ} \mathrm{C}$ for $30 \mathrm{~min}$, producing clean atomically flat terraces with a typical width of $60 \mathrm{~nm}$.

\section{FLUCTUATION MEASUREMENTS}

\section{A. Frequency spectrum of fluctuations}

In order to characterize the dynamic properties of the cantilever force sensor, we have measured fluctuations in the lateral force signal. In Fig. 1(a), the power spectral density of the signal is compared for the free cantilever and for the cantilever with the sharp tip resting in contact with the $\mathrm{KBr}$ surface. The sharpness of the peak at $168.6 \mathrm{kHz}$ reflects the high quality factor of $Q_{\text {free }} \sim 350000$ for this oscillation mode. When the tip is in contact, the resonance frequency shifts to $172.5 \mathrm{kHz}$, and its width increases significantly. Assuming a harmonic oscillator model to be adequate, the width corresponds to a $Q$-factor of $Q_{\text {contact }} \sim 700$. An additional ring-down measurement for an externally excited oscillation $^{8}$ confirms a quality factor of about 600 . This frequency spectrum of the lateral force signal does not change significantly when the tip is scanned over the $\mathrm{KBr}(100)$ surface. The only additional features are a peak at a low frequency given by the periodicity of the stick-slip events, and the higher harmonics of this frequency reflecting the sawtooth shape of the stick-slip signal.

\section{B. Load dependence of the resonance}

The occurrence of stick-slip instabilities and accordingly of energy dissipation in atomic friction experiments depends critically on the normal load on the contact. ${ }^{2}$ However, once the contact was established we found no monotonous dependence of the position or width of the torsional resonance peak in Fig. 1(a) depending on loads up to $15 \mathrm{nN}$ applied to the tip. The position of the resonance peak varies as much as $700 \mathrm{~Hz}$ even for subsequent measurements at the same load. Any distinct relation between resonance and load is hidden by this fluctuation. This result is in some contrast to findings by Drobek et al. ${ }^{9}$ who reported that for a $v$-shaped cantilever in air the resonance frequency of the first torsional mode increases with higher loading forces and related the increase of frequency to an increase of the contact stiffness. In order to elucidate whether the vacuum environment or the different tip sample interaction cause the differences between our and Drobek's findings we have recorded the thermal fluctuation spectra for spherical tips with micron-scale apex radius. Upon contact to the flat $\mathrm{Al}_{2} \mathrm{O}_{3}(0001)$ surface, frequency and quality factor change in the same sense as when the sharp tip on the $\operatorname{KBr}(100)$ comes in contact with surface. Upon increasing the normal load, the frequency shift increases as seen in Fig. 1(b). With increasing load, the width of the resonance curves decreases and its peak height increases. Using a harmonic oscillator analysis this would imply that the quality factor in contact increases with increasing load. The total area below the peaks is reduced by the increasing load. Therefore, the description of the curves as thermal fluctuations in a point-mass model is not sufficient.

\section{DETERMINATION OF SLIP DURATION}

Figure 2(a) shows a typical friction loop, where the lateral force for a single scan line in forward and backward direction is measured. All stick-slip friction loops were recorded on a $\mathrm{KBr}(100)$ surface along the (001) direction when the tip was moving through the center of the apparent unit cells, i.e., along lines of maximal modulation of the lateral force. The sawtooth-shape signal consists of sticking phases where the 

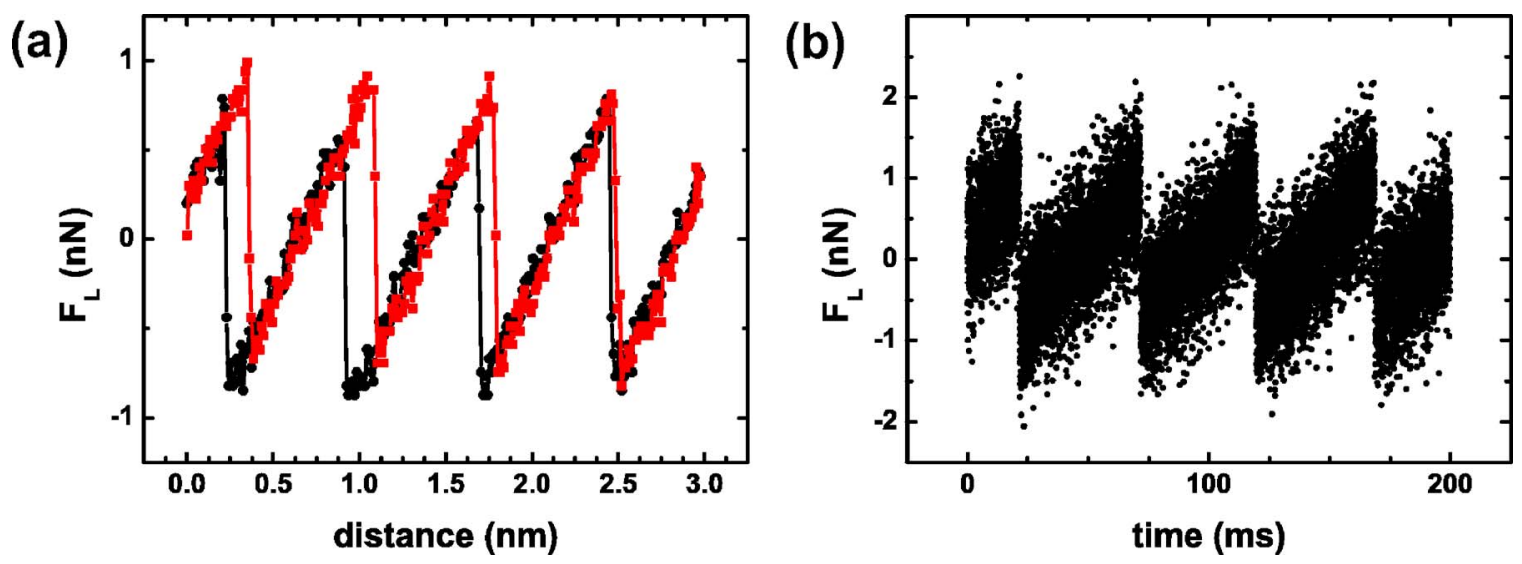

FIG. 2. (Color online) (a) Friction force loop recorded as part of a typical scan frame on a $\mathrm{KBr}(100)$ crystal along the (001) direction. The time resolution is typical for commercially available data acquisition setups. The cantilever in this experiment is the same as the one used to produce the data for Fig. 1(a). (b) High temporal resolution scan line simultaneously acquired with the forward scan data [square symbol in (a)] at a sampling frequency of 3.3 MHz, subsequently averaged over 50 points in order to clearly reveal the shape of the stick-slip pattern.

lateral force is built up in an almost linear slope, and slip events where the tip jumps from one atomic position to the next. The slope of the sticking part, $k_{\text {exp }}$, reflects the effective lateral stiffness of the force sensor and the contact. Here, we find $k_{\text {exp }}=2.23 \pm 0.05 \mathrm{~N} / \mathrm{m}$. The data for this figure was acquired with a commercial data acquisition setup (SCALA, OMICRON Nanotechnology). The sampling rate of data points recorded $(1250 \mathrm{~Hz}$ at scan velocity of $15 \mathrm{~nm} / \mathrm{s})$ is not high enough either to detect thermal fluctuations in the force signal or to measure the duration of each slip. Therefore, we simultaneously acquired the lateral force signal with a sampling frequency of $3.3 \mathrm{MHz}$, the bandwidth of our force detection. The actual stick-slip structure can be made out in a wideband of noise, composed of both thermal fluctuations of the cantilever and electronic noise. In order to analyze the slip duration we have averaged the force data over 50 points so that the stick-slip characteristics can be clearly recognized, reducing the bandwidth of the results to $66 \mathrm{kHz}$ or to a time resolution of $15 \mu \mathrm{s}$. A typical scan line in forward direction is shown in Fig. 2(b). A tilted step function is fit to the averaged data in order to determine the slip duration. Figure 3 shows four averaged curves. The same instrumental parameters were used for the measurements: normal load $F_{N}=-0.1 \mathrm{nN}$ and scan velocity $v=25 \mathrm{~nm} / \mathrm{s}$. We extract a slip duration of $t \approx 8.2 \mathrm{~ms}$ in Fig. 3(a) and $t<15 \mu \mathrm{s}$ in Fig. $3(\mathrm{~b})$. The duration of the second slip is below the limit of time resolution and could actually even be shorter than $15 \mu \mathrm{s}$. As a first conclusion we find that the slip process in atomic friction can happen on very different time scales for similar experimental conditions.

Figures 3(c) and 3(d) give examples of slip events which suggest possible mechanisms for the prolonged slip duration. In Fig. 3(c) the tip jumps forth and back between two atomic positions before finally settling at the new position. In Fig. 3(d) the tip jumps into an intermediate position before the jump over a full lattice constant is finished.

An overview of the distribution of slip durations for several tips which range from some microseconds up to several milliseconds is given in Fig. 4. We have included corresponding lateral force maps in order to reveal possible cor- relations between the appearance of such maps and the duration of slip events. Lateral force maps corresponding to fast slips appear as a pattern of flat tilted diamonds with the size of one unit cell of the $\mathrm{KBr}(100)$ surface. In contrast, maps associated with slower slips show features on a scale smaller than the unit cell. Such features could arise from an irregular structure of the tip. Several atoms of the tip may be in contact with different unit cells of the surface, having a distance between the contact points that is not in registry with the $\operatorname{KBr}(100)$ surface structure.

\section{SIMULATION OF THE STICK-SLIP PROCESS}

A model comprised of a single spring pulling a mass along a sinusoidal potential surface which included thermal activation has described atomic friction phenomena like temperature and velocity dependence, and the distribution of jump forces. ${ }^{5}$ The results are in agreement with experiments studying the velocity dependence, ${ }^{4}$ the temperature dependence, ${ }^{10}$ and the jump force distribution. ${ }^{11}$ However, the experimental situation is not well represented, since a one-spring model cannot reproduce both the resonance frequency of the cantilever, and the experimentally found lateral stiffness which is dominated by the contact. Therefore we extend the simulations to a two-spring model, where one spring represents the cantilever and the other spring the microscopic contact. The two springs are connected in series to a support moving at constant velocity. A tip attached to the contact spring is dragged over a periodic potential surface [see Fig. 5(b)]. Including the random effect of thermal fluctuations we have simulated the resulting Langevin equation using Ermak's algorithm ${ }^{12}$ along the lines pointed out in Ref. 5.

The parameters describing the cantilever spring in the two-spring model are taken from the experimental data. An effective cantilever mass $m_{c l}=k_{T} /\left(2 \pi f_{T}^{f r e e}\right)^{2}=5.5 \times 10^{-11} \mathrm{~kg}$ can be calculated using the frequency of the free cantilever $f_{T}^{f r e e}=168.6 \mathrm{kHz}$ and the spring constant $k_{T}=62 \mathrm{~N} / \mathrm{m}$.

The occurrence of the stick-slip instability in the tip movement depends on the relation between the potential cor- 
(a)

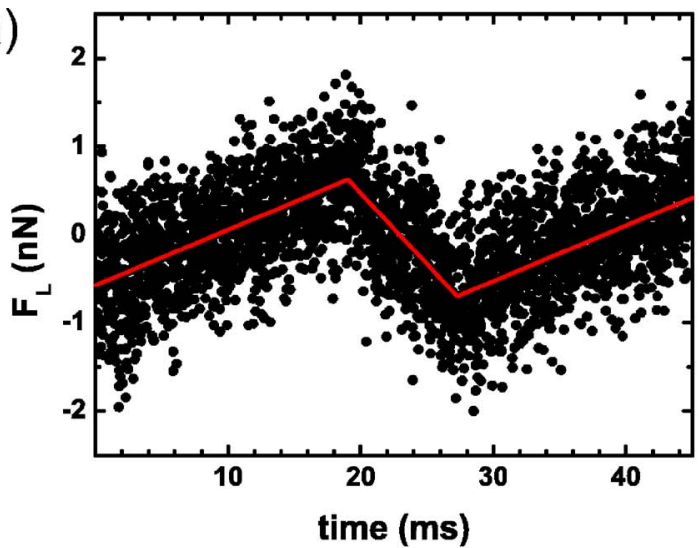

(c)

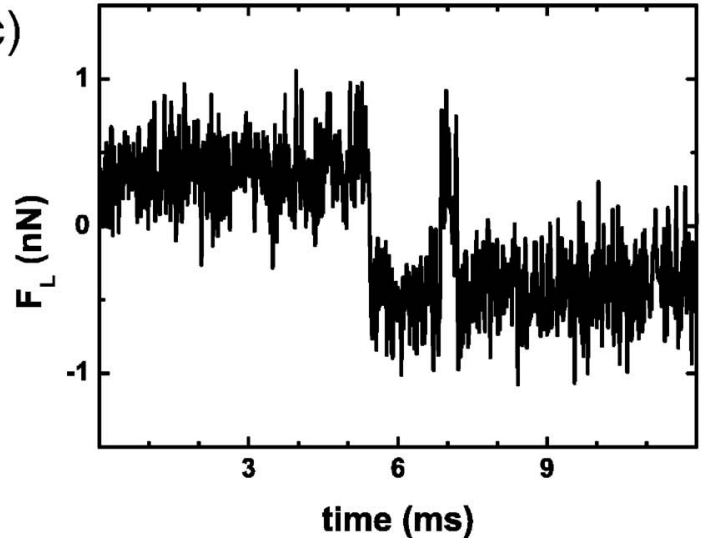

(b)

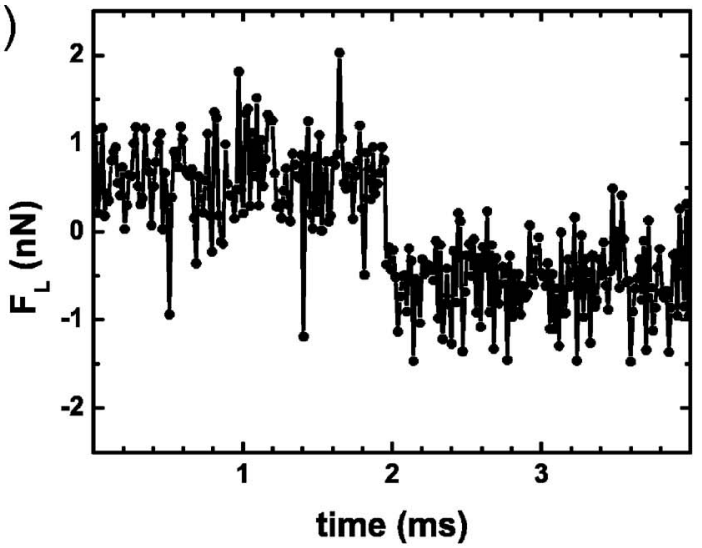

(d)

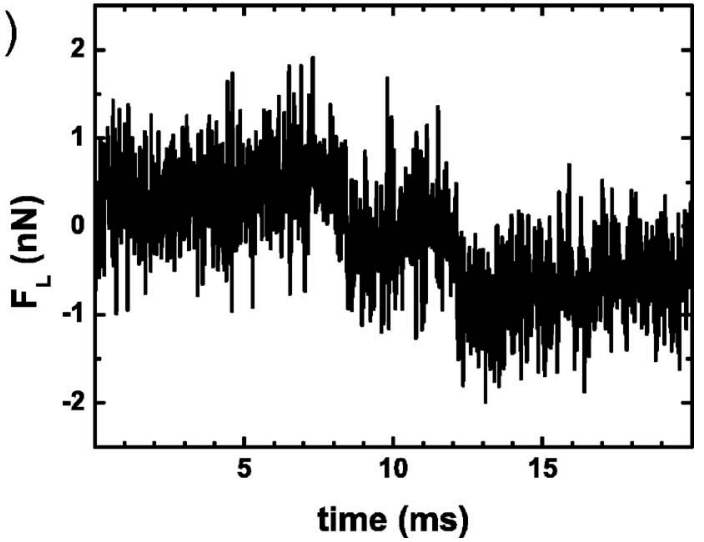

FIG. 3. (Color online) Lateral force vs time acquired on $\operatorname{KBr}(100)$ with a sampling frequency of $3.3 \mathrm{MHz}$ and subsequently averaged over 50 data points. The same scan parameters were used for these four measurements of atomic stick-slip: normal force $F_{N}=-0.1 \mathrm{nN}$ and scan velocity $v=25 \mathrm{~nm} / \mathrm{s}$. The slip duration was determined to be (a) $t \approx 8.2 \mathrm{~ms}$ and (b) $t<15 \mu \mathrm{s}$. (c) In the process of slipping to the next atomic position, the tip jumps forth and back between the new and the old position. (d) During the slip, the tip assumes an intermediate position.

rugation $E_{0}$ and the elastic energy in the springs. It can be described by the parameter

$$
\eta=\frac{2 \pi^{2} E_{0}}{k_{s} a^{2}} \quad \text { with } \quad \frac{1}{k_{s}}=\frac{1}{k_{T}}+\frac{1}{k_{\text {tip }}} .
$$

Stick-slip behavior is found for $\eta>1 .^{2}$ We choose $k_{s}$ $=3.7 \mathrm{~N} / \mathrm{m}$ and a value of $\eta=2$ in order to obtain a value for the potential corrugation $E_{0}$ for which the simulation produces a stick-slip pattern similar to the experimental results.

Equation (1) allows us to determine $k_{t i p}=3.5 \mathrm{~N} / \mathrm{m}$. The lattice constant of $\operatorname{KBr}(100) a=0.66 \mathrm{~nm}$ determines the spatial period of the potential. The mass of the tip is assumed to be $m_{t i p}=1.0 \times 10^{-12} \mathrm{~kg}$. Since we have no experimental estimate for the effective mass of the tip which corresponds to the tip stiffness, we have varied $m_{t i p}$ by a factor of ten for the simulations, and found no influence on the key results described below. The damping of the tip movement is calculated in units of the critical damping $\gamma_{c}=2 \sqrt{k_{t i p} / m_{\text {tip }}}$. Reimann and Evstigneev have suggested to model tip damping based on the tip velocity relative to the surface but also relative to the support. ${ }^{6,7}$ For the low sliding velocities simulated here such distinction is not necessary. The simulations were performed at room temperature and for a scan velocity of $25 \mathrm{~nm} / \mathrm{s}$. The time step used for solving the Langevin equa-

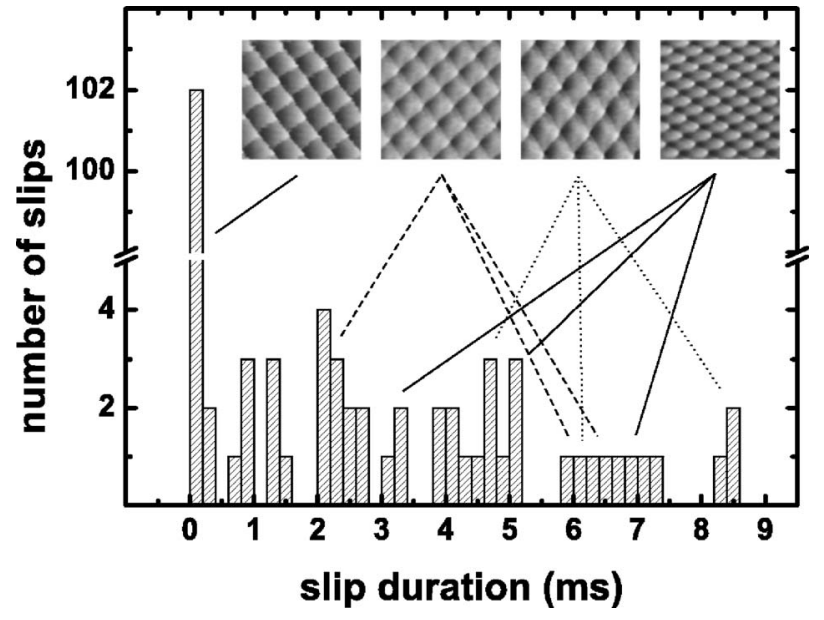

FIG. 4. Distribution of the slip durations for 150 slip measurements on $\mathrm{KBr}(100)$ taken from 46 different scan frames using four different tips. The applied load was always very close to $0 \mathrm{nN}$. Four lateral force maps are shown to reveal possible correlations between their appearance and the duration of slip events. Note the irregular features within the unit cell for the three maps on the right. The lines indicate which slip durations were found in which lateral force map. The bar for shortest slip duration reflects the slips which happened faster than our time resolution of $15 \mu$ s. 


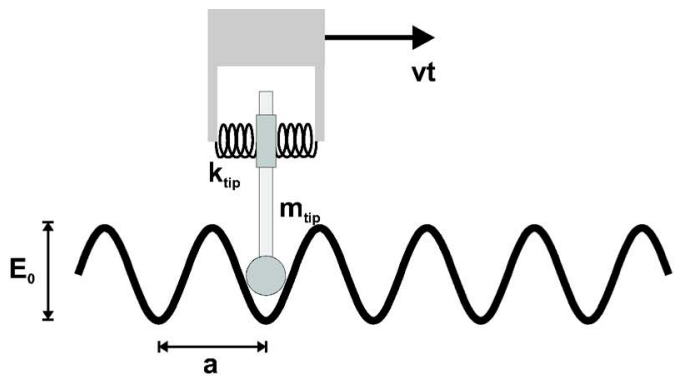

(a)

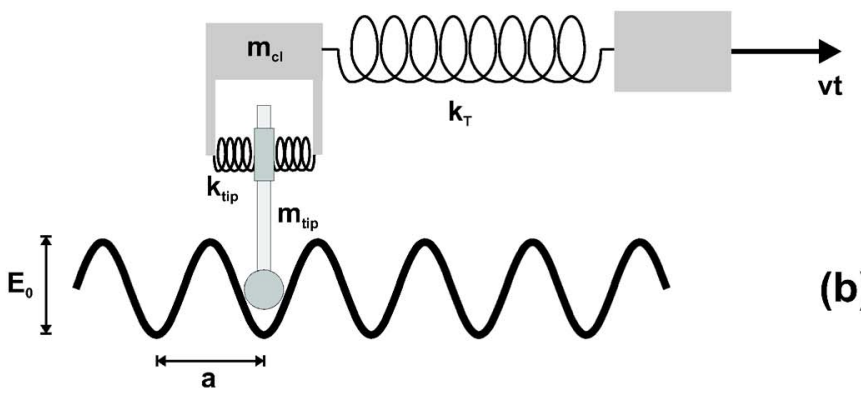

FIG. 5. (Color online) Three spring configurations for different implementation of the Tomlinson model.

(b)

(c)

tion was $1 \mathrm{~ns}$, data points were sampled from the simulation at a rate of $3 \mathrm{MHz}$ equal to the experiment.

Simulations using the two-spring model and the parameters described above have provided the expected stick-slip results, plotted in Fig. 6(a). However, the slip duration was always shorter than $15 \mu$ s, independent of the damping of the contact spring which was varied from critical to strong damping.

Experimental friction maps often show features within a unit cell which let us conclude that the contact between the tip and the surface consists of several smaller contacts. Therefore, we expanded the two-spring model to the case of multiple tips in contact with the potential surface, where the distance between tips was either commensurate or incommensurate with the periodicity of the potential. Figure 5(c) gives a scheme of the multiple-tip model. The parameters for each $N$-tip simulation were the same as described above, with each of the contact springs having a tip stiffness of $k_{t i p} / N$ and a mass of $m_{t i p} / N$. We also divided the potential corrugation $E_{0}$ by the number of tips $N$. This scaling is chosen in order to always reproduce the experimentally observed slope and amplitude of the stick-slip force curves, however, for a different number of tips involved.

For the multiple-tip model, the dynamics of each tip $i$ is described by the Langevin equation

$$
m_{t i p} \ddot{x}_{t i p, i}+m_{t i p} \gamma_{t i p} \dot{x}_{t i p, i}+\frac{\partial E\left(x_{t i p, i}\right)}{\partial x}=\xi(t),
$$

where the combined surface-tip potential has the form

$$
E\left(x_{t i p, i}\right)=\frac{k_{t i p}}{2}\left(x_{t i p, i, 0}-x_{t i p, i}\right)^{2}-\frac{E_{0}}{2} \cos \left(\frac{2 \pi x_{t i p, i}}{a}\right) .
$$

$x_{t i p, i, 0}$ is the equilibrium position where the springs $k_{t i p}$ have no deviations. $\xi(t)$ is a random noise satisfying the fluctuation-dissipation relation $\left\langle\xi(t) \xi\left(t^{\prime}\right)\right\rangle=2 m_{t i p} \gamma_{t i p} k_{B} T \delta(t$ $-t^{\prime}$ ), where the angular brackets denote an average, and $k_{B}$ is Boltzmann's constant.

As the mass of the cantilever is many orders of magnitude larger than the tip's, the thermal effects on the cantilever can be neglected. Therefore, the cantilever's motion is described by Newton's equation

$$
m_{c l} \ddot{x}_{c l}+m_{c l} \gamma_{c l} \dot{x}_{c l}-k_{c l}\left(v t-x_{c l}\right)+\sum_{i} k_{t i p}\left(x_{t i p, i, 0}-x_{t i p, i}\right)=0 .
$$

Figure 6 shows simulation results for the stick-slip process with a multiple-tip model for critical damping of the contact springs. We compare the cases of one, three, and five tips, where the multiple tips are in positions commensurate with the potential surface. The number of tips is crucial for the duration of the slip: The slips become slower for an increasing number of tips. As mentioned above, a single tip always slips faster than $15 \mu \mathrm{s}$. For five tips we observe slip durations in the range of $2.8 \mathrm{~ms}-3.2 \mathrm{~ms}$. For three tips, the slips show a wide variation of duration between microseconds and milliseconds. In this case the slip often contains intermediate states, where one or several tips have jumped 

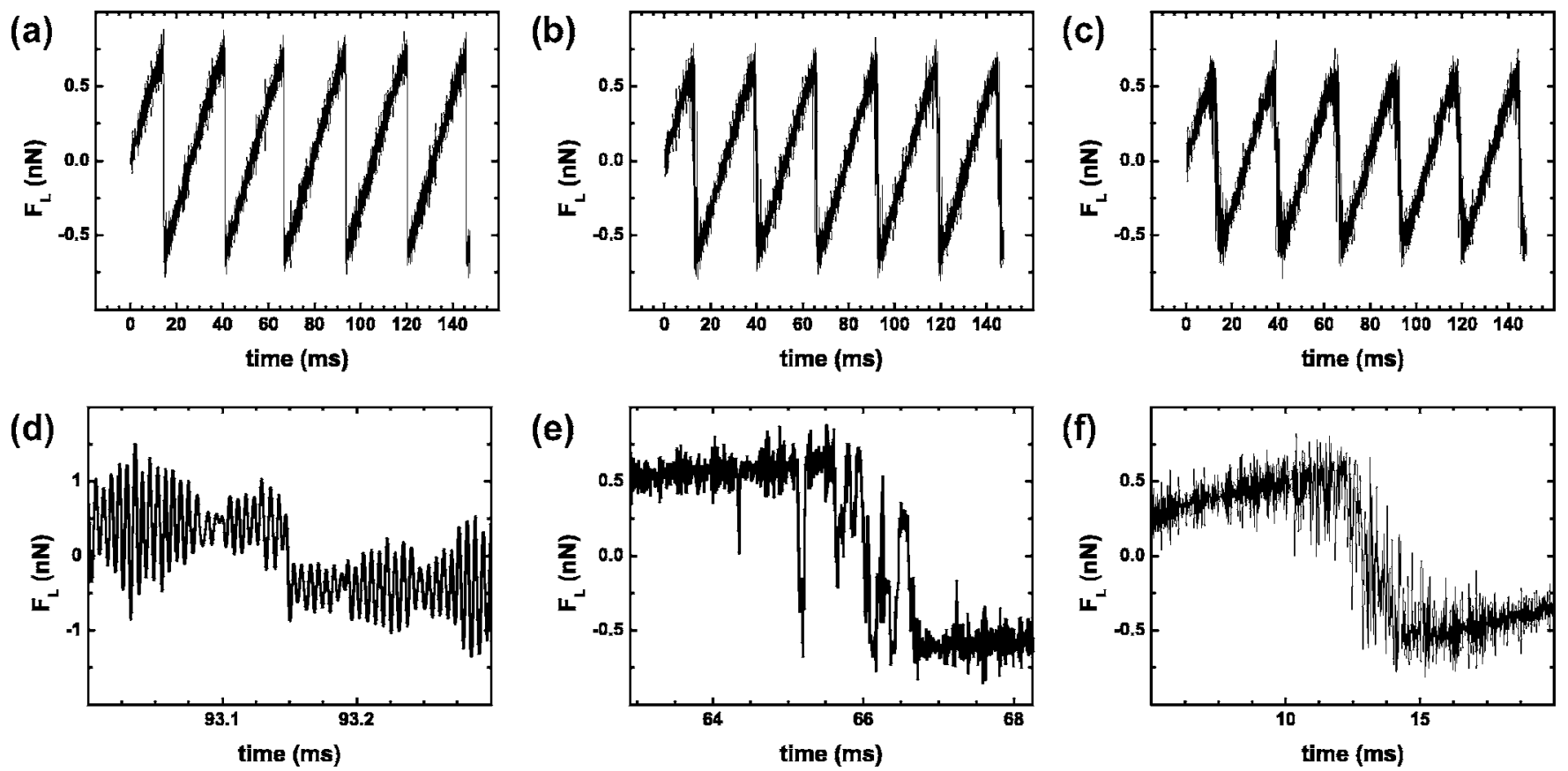

FIG. 6. Simulation of the stick-slip process with a critically damped two spring model. The contact consists of (a) one, (b) three, and (c) five commensurate tips. Data were sampled from the simulation at a rate of $3 \mathrm{MHz}$ and subsequently averaged over 50 points. (d) Detail of the simulation with one tip, no averaging. (e) and (f) Details of the simulation for three and five tips, respectively, averaged over 30 points.

but others still stick. The duration of the slip does not depend significantly on the damping of the contact springs; no difference was noticed for critical damping and the ten times overdamped system. Details of slip events are plotted in Figs. 6(d)-6(f). For the one-tip model, the slip happens faster than the data capture rate of $3 \mathrm{MHz}$. It is interesting to note that the thermal fluctuations clearly follow the frequency of the cantilever resonance. However, no excitation of oscillations with subsequent ring-down can be observed after jumps. The simulation for three tips reveals jumps forth and back between the previous and the final position and into intermediate states, indicating the mechanisms responsible for the wide variation of slip durations. For five tips, multiple events of tip jumps smoothen the transition from one atomic position to the next and cause the prolonged slip duration.

We have also studied the case of multiple tips in incommensurate contact with the potential surface, not shown here. For a small number of tips with a distance of $1.1 \times a$ the periodicity of the potential can be recognized in the lateral force. However, for as few as five tips the asymmetric stickslip instabilities are almost completely changed into a symmetric force modulation. For a fully incommensurate configuration with a tip distance $\sqrt{2} \times a$ no structure of the potential is reproduced in the lateral force.

\section{DISCUSSION}

We start the discussion with an analysis of changes in the resonance structure of the cantilever's thermal fluctuations upon contact of the tip with the surface. First of all, the frequency of the torsional resonance of the cantilever increases upon contact formation. The increase can be ex- plained by the additional stiffness caused by the fixation of the tip apex. It has been observed in ambient environment and described in a finite element analysis by Drobek et al. who used such frequency shift to determine the shear stiffness of the contact. ${ }^{9}$ For rectangular cantilevers, a point-mass harmonic oscillator model can describe the frequency shift reasonably well. The resonance frequency of the torsion mode of the cantilever can be modeled as

$$
f_{T}^{\text {free }}=\frac{1}{2 \pi} \sqrt{\frac{k_{T}}{m_{c l}}}
$$

where $k_{T}$ is the spring constant for torsional bending and $m_{c l}$ is an effective mass of the cantilever. Bringing the tip in contact, the contact stiffness $k_{\text {con }}$ can be added to the spring constant and

$$
f_{T}^{c o n}=\frac{1}{2 \pi} \sqrt{\frac{k_{T}+k_{c o n}}{m_{c l}}} .
$$

The contact stiffness, a combination of the tips stiffness and the curvature of the surface potential, can be calculated from the slope of the force curve $k_{\text {exp }}$ in Fig. 2(a) assuming that the cantilever and the contact act as springs in series

$$
\frac{1}{k_{\text {con }}}=\frac{1}{k_{\text {exp }}}-\frac{1}{k_{T}} .
$$

Combining Eqs. (6) and (7), the point-mass spring model predicts 


$$
f_{T}^{\text {con }}=f_{T}^{\text {free }} \sqrt{1+\frac{1}{\frac{k_{T}}{k_{\text {exp }}}-1}} .
$$

With $k_{\exp }=2.23 \pm 0.05 \mathrm{~N} / \mathrm{m}$ from Fig. 2 we expect $f_{T}^{\text {con }}$ $=171.75 \pm 0.08 \mathrm{kHz}$, a little less than the actual value of $172.5 \mathrm{kHz}$ found in Fig. 1. The discrepancy can easily be caused by the error in the determination of $k_{T}$, which depends critically on the precise measurement of the actual height of the tip. Another explanation for the discrepancy could be a change of the effective mass $m_{c l}$ if the shape of the cantilever deflection is modified by the contact formation. However, considering the small relation between contact stiffness $k_{c o n}$, cantilever stiffness $k_{T}$, and the small thermal oscillation amplitude of about one tenth of an atomic diameter at the tip apex, a significant change of the deflection mode can be excluded.

A further frequency shift with increasing load on the contact is found for micrometer-sized spherical tips. For the sharp tips used in atomic friction experiments, no clear tendency of the frequency shift is observed, but a smaller fluctuation of the peak position even for measurements at the same load. Following the point-mass model or the model of Drobek et al. and taking the frequency shift as a measure for the contact stiffness, ${ }^{9}$ we find that the contact stiffness for very sharp tips is not load dependent but shows smaller variations as a function of time. This is in agreement with prior analysis of the load dependence of the contact stiffness from atomic friction data. ${ }^{2}$ In a continuum mechanics approach we expect the lateral stiffness to increase with load proportional to the contact area. The breakdown of continuum models at the nanometer scale for the contact stiffness has recently been discussed by Luan and Robbins, who found that their applicability depends on the atomic structure of the tip apex. Tips that maintain a spherical shape even on nanometer scale are well described by continuum models, while tips with a stepped atomic structure exhibit discontinuities in the contact area with increasing load. ${ }^{13}$ We find apex clusters with a stepped structured a more probable tip model and conclude from our results that for very sharp tips and low loads the contact size and the contact stiffness do not vary as a function of load. The unsystematic changes of the contact stiffness are related to changes of the contact geometry, due to uncontrolled processes such as diffusion or drift. For larger spherical tips and higher loads, our data in Fig. 1(b) confirm the established relations between load, contact size, contact stiffness, and shift in the torsional resonance frequency. We have determined an increasing lateral contact stiffness from the slope in friction loops reaching $k_{\text {exp }}=32 \mathrm{~N} / \mathrm{m}$ for the highest load of $F_{N}=51 \mathrm{nN}$, i.e., the lateral contact stiffness $k_{s}$ becomes equal to the cantilever stiffness $k_{T}$.

Upon contact formation, we do not only observe a shift of the resonance frequency but also a drastic increase of the width of the resonance peak, corresponding to a drop in the quality factor by nearly three orders of magnitude. This observation is only possible in vacuum, as in earlier studies in ambient environment the quality factor is dominated by air damping. Let us consider possible sources of the additional dissipation. The quality factor of the free cantilever is given by the internal friction in the beam. As discussed above, a significant change of the shape of the cantilever deflection upon contact formation can be excluded. Consequently, the observed strong damping cannot be caused by additional internal friction in the cantilever. Rather, dissipation in the tipsample contact causes the decrease in the quality factor of the cantilever. In order to analyze how damping of the tip deflection will influence the cantilever motion we calculate the quality factor of the cantilever oscillation for the twospring model depicted in Fig. 5(b). The tip motion is described by Newton's equation

$$
m_{t i p} \ddot{x}_{t i p}=-k_{t i p}\left(x_{t i p}-x_{c l}\right)-m_{t i p} \gamma_{t i p} \dot{x}_{t i p}
$$

where $x_{t i p}$ and $x_{c l}$ are the respective deflections of tip and cantilever, $\gamma_{t i p}$ is a damping coefficient for the tip movement, and the other parameters are the same as in Sec. V. We neglect the internal friction of the cantilever since we know that dissipation due to the tip motion dominates. Assuming for this derivation that the cantilever oscillates with $x_{c l}$ $=A_{c l} \sin \left(\omega_{c l} t\right)$, with $\omega_{c l}=\sqrt{k_{T} / m_{c l}}$, the equation of motion [Eq. (9)] becomes

$$
\ddot{x}_{t i p}+\gamma_{t i p} \dot{x}_{t i p}+\omega_{t i p}^{2} x=\frac{k_{t i p} A_{c l}}{m_{t i p}} \sin \left(\omega_{c l} t\right) .
$$

The time-averaged power loss in this driven, weakly damped oscillation of the tip is given as

$$
\bar{P}=\frac{k_{t i p}^{2} A_{c l}^{2} \gamma_{t i p}}{2 m_{t i p}} \frac{\omega_{c l}^{2}}{\left(\omega_{t i p}^{2}-\omega_{c l}^{2}\right)^{2}+\omega_{c l}^{2} \gamma_{t i p}^{2}} .
$$

This dissipation in the tip movement will damp the cantilever oscillation. The quality factor $Q_{c o n}$ of the cantilever is defined as the relation between the total energy of its oscillation and the power loss per oscillation cycle

$$
Q_{c o n}=2 \pi \frac{E}{\Delta E}=2 \pi \frac{\frac{1}{2} k_{T} A_{c l}^{2}}{2 \pi \frac{\bar{P}}{\omega_{c l}}}=\frac{1}{2} \frac{k_{T} A_{c l}^{2} \omega_{c l}}{\bar{P}} .
$$

Combining Eqs. (11) and (12) and introducing the critical damping $\gamma_{c}=2 \omega_{\text {tip }}$ for the tip movement we obtain

$$
Q_{c o n}=\frac{1}{2}\left(\frac{m_{t i p}}{m_{c l}}\right)^{1 / 2}\left(\frac{k_{T}}{k_{t i p}}\right)^{3 / 2}\left(\frac{\gamma_{c}}{\gamma_{t i p}}\right)\left[\left(\frac{\omega_{t i p}}{\omega_{c l}}-\frac{\omega_{c l}}{\omega_{t i p}}\right)^{2}+4 \frac{\gamma_{t i p}^{2}}{\gamma_{c}^{2}}\right] \text {. }
$$

For the parameters used in the simulations, $\omega_{\text {tip }}$ $=\sqrt{k_{\text {tip }} / m_{\text {tip }}}$ and $\gamma_{\text {tip }}=\gamma_{c}$, one obtains $Q_{\text {con }} \sim 30$, in the same order as the result for a resonance analysis of the simulated lateral force data. For $m_{\text {tip }} \ll m_{c l}$ we have $\omega_{\text {tip }} \gg \omega_{c l}$ and the expression simplifies into

$$
Q_{\text {con }}=\frac{1}{2}\left(\frac{m_{c l}}{m_{\text {tip }}}\right)^{1 / 2}\left(\frac{k_{T}}{k_{\text {tip }}}\right)^{1 / 2}\left(\frac{\gamma_{c}}{\gamma_{\text {tip }}}\right) .
$$

As one would expect, the quality factor $Q_{c o n}$ of the cantilever is lowered by the effective mass $m_{\text {tip }}$ of the tip, the coupling $k_{t i p}$ between cantilever and tip, and by the damping 
$\gamma_{\text {tip }}$ of the tip movement. In order to reach a quality factor of the order of 600 as found in the experiment, one would have to assume a hundred times smaller tip mass or a strong underdamped tip movement. No signatures of the latter has been observed in experiments. On the other hand, an effective tip mass of only $m_{\text {tip }}=10^{-14} \mathrm{~kg}$ would make sense as it corresponds to a volume of $1 \mu \mathrm{m} .{ }^{3}$ Reimann and Evstigneev have come to a similar conclusion in their analysis of friction force microscopy focusing onto the tip deflection. ${ }^{7}$ The authors have also employed a two-spring model where the main physical effects of atomic friction and dissipation are attached to the contact spring, while the cantilever spring serves as a detection tool.

With a quality factor of 600 for the cantilever torsion one may expect to observe a ringdown of the oscillation after fast slip events. The excitation of such oscillations by each slip event has been reported for an uncalibrated atomic friction experiment on graphite. ${ }^{14}$ The cited experiment is difficult to compare to our results since the magnitude of forces is unknown and the experimental method included bandpass filtering around the expected torsional resonance. In both our experimental and simulation results we do not observe any excitation of cantilever oscillations by the slip events. The magnitude of the force relaxation in the slip is of the same order or smaller than the thermal fluctuations of the force signal. Therefore, excitations of oscillations with subsequent ring-down cannot be observed since thermal oscillations dominate.

The increase of the quality factor with increasing load observed for the micrometer-sized contact and higher loads cannot be explained in a simple two-spring model. The lateral contact stiffness becomes comparable to the torsional stiffness of the cantilever. Consequently, the contact can act as a hinge and change the shape of the cantilever deflection, as can be inferred from the changing area under the resonance curves in Fig. 1(b). More complex models including the finite volume of the beam and internal friction following stress distributions will be necessary to predict the quality factor in these cases.

As a result of the analysis of the fluctuation spectra we can summarize that there must be a strong damping of the tip movement mediated by the tip-sample contact. The observed reduction of the quality factor of the cantilever movement due to the tip damping can be coherently described using a two-spring model, as long as the contact stiffness is small compared to the stiffness of the cantilever.

We will now discuss the wide variation of slip durations in the atomic stick-slip experiments. The shortest possible duration of a slip is given by the time the cantilever needs to swing back from the deflected to the relaxed position, i.e., $1 /\left(4 f_{T}\right)$. With $f_{T}^{c o n} \approx 172 \mathrm{kHz}$ this reaction time is $1.5 \mu \mathrm{s}$. While we observe that many slips happen indeed on a time scale faster than our time resolution of $15 \mu \mathrm{s}$, other slips may take up to several milliseconds. These long slip are very surprising as we would not expect any relaxation process on atomic scale to be that slow.

A possible explanation for slow slip events can be found in the framework of the Tomlinson model. The effective potential governing the tip movement consists of a sinusoidal surface potential and a parabolic spring potential which are continuously shifted relative to each other. The tip is stuck in a potential minimum until the ramped spring potential flattens the potential barrier to the next atomic position. The decrease of the resonance frequency of the system when approaching the slip instability is described in Refs. 5 and 15. When the resonance frequency decreases in the flattened potential a slower reaction of the cantilever might be expected. On the other hand, thermal fluctuations activate the slip before the potential barrier has completely vanished, resulting again in a fast slip. The simulation results in Figs. 6(a) and 6(d) demonstrate that no retardation of the slip takes place in the single-contact model. All slip durations are clearly faster than the resolution of $15 \mu \mathrm{s}$. As a conclusion, we can rule out any explanation for long slip durations within the simple one-dimensional Tomlinson model including thermal activation at room temperature.

Our results suggest that multiple tips in contact play an important role for the understanding of slow slip events. We measure fast slips in the order of several microseconds, if the lateral force maps look similar to the two-dimensional modeling for an ideal single tip reported by Lüthi et al. ${ }^{16}$ Slow slips are encountered when the lateral force maps exhibit features within a lattice unit cell or deviate significantly from the expected diamond pattern, see Fig. 4. Such irregularities can be explained only by multiple tips which are in contact with different unit cells of the $\mathrm{KBr}$ surface. The simulations support the idea that multiple tips can give a clear stick-slip motion, however with a significantly longer slip duration than the reaction time of the force sensor. Our three-tip model with thermal activation also reproduces the wide distribution of slip durations found in the experiments. Furthermore, the simulations indicate mechanisms for the retardation of the slips. Figure 6(e) shows that multiple tips do not jump coherently but that single tips can jump ahead and actually may go back to their previous atomic position before the increasing lateral force enforces a jump of all tips. A similar mechanisms of molecular rebinding has been predicted through simulations of the rupture of adhesion bonds in dynamic force spectroscopy by Dudko et al. ${ }^{17}$ In recent theoretical study, Krylov et al. have pointed out that the thermally activated jumps between atomic positions can create a situation of ultralow friction, provided that the scan velocity is small enough. ${ }^{18}$ The jump mechanism is clearly observed in our experiments. Figures 3(c) and 3(d) exhibits signatures of jumps between positions and intermediate states, respectively. The latter may also be an effect of two-dimensional stick-slip where the slipping tip takes a zigzag path including a neighboring unit cell. ${ }^{19,20}$ However, a zigzag movement should produce its characteristic slip pattern for all slip in a given scan line. Since we observe a wide distribution of slip times and shapes in a single scan line, we believe that thermally activated jumps of a multiple tip are the appropriate picture for our experiments.

\section{CONCLUSION}

Atomic friction phenomena have been studied in detail by means of a home-built force microscope allowing for highbandwidth detection of the lateral force signal. Thermal fluc- 
tuations of the cantilever which have a strong impact on the atomic stick-slip process are strongly damped through the tip-sample contact. By modeling the experiment by two springs, one representing the cantilever force sensor and one the compliant tip-sample contact, a consistent description of the lateral contact stiffness in static and dynamic lateral force measurements has been achieved. Recording the atomic stick-slip movement of the force microscope tip with high bandwidth reveals a wide variation of slip durations up to several milliseconds, by far longer than expected for a relaxation process on atomic scale. These long slip events are believed to be the consequence of a multiple contact between tip and surface. This conclusion is drawn from a correlation between irregular features in lateral force maps and long slip durations, and from a comparison of our experimental results with a multi-tip simulation based on a Tomlinson model including thermal activation.

\section{ACKNOWLEDGMENTS}

The McGill group is indebted to Stephan Messmer, HansRudolf Hidber, and Andreas Tonin of the University of Basel for support in building the experimental setup. This work was supported by the Canada Foundation of Innovation, the NSERC, and the Swiss National Science Foundation.
*Permanent address: Department of Physics and Astronomy, University of Basel.

$\dagger$ Electronic address: roland.bennewitz@mcgill.ca

${ }^{1}$ C. M. Mate, G. M. McClelland, R. Erlandsson, and S. Chiang, Phys. Rev. Lett. 59, 1942 (1987).

${ }^{2}$ A. Socoliuc, R. Bennewitz, E. Gnecco, and E. Meyer, Phys. Rev. Lett. 92, 134301 (2004).

${ }^{3}$ M. Dienwiebel, G. S. Verhoeven, N. Pradeep, J. W. M. Frenken, J. A. Heimberg, and H. W. Zandbergen, Phys. Rev. Lett. 92, 126101 (2004).

${ }^{4}$ E. Gnecco, R. Bennewitz, T. Gyalog, C. Loppacher, M. Bammerlin, E. Meyer, and H. J. Güntherodt, Phys. Rev. Lett. 84, 1172 (2000).

${ }^{5}$ Y. Sang, M. Dube, and M. Grant, Phys. Rev. Lett. 87, 174301 (2001).

${ }^{6}$ P. Reimann and M. Evstigneev, Phys. Rev. Lett. 93, 230802 (2004).

${ }^{7}$ P. Reimann and M. Evstigneev, New J. Phys. 7, 25 (2005).

${ }^{8}$ O. Pfeiffer, C. Loppacher, C. Wattinger, M. Bammerlin, U. Gysin, M. Guggisberg, R. Bennewitz, E. Meyer, and H.-J. Güntherodt, Appl. Surf. Sci. 157, 337 (2000).

${ }^{9}$ T. Drobek, R. W. Stark, and W. M. Heckl, Phys. Rev. B 64,
045401 (2001).

${ }^{10}$ S. Sills and R. M. Overney, Phys. Rev. Lett. 91, 095501 (2003).

${ }^{11}$ A. Schirmeisen, L. Jansen, and H. Fuchs, Phys. Rev. B 71, 245403 (2005).

${ }^{12}$ M. Allen and D. Tildesley, Computer Simulations of Liquids (Clarendon, Oxford, 1990).

${ }^{13}$ B. Luan and M. Robbins, Nature (London) 435, 929 (2005).

${ }^{14}$ T. Kawagishi, A. Kato, Y. Hoshi, and H. Kawakatsu, Ultramicroscopy 91, 37 (2002).

${ }^{15}$ A. Garg, Phys. Rev. B 51, 15592 (1995).

${ }^{16}$ R. Lüthi, E. Meyer, M. Bammerlin, L. Howald, H. Haefke, T. Lehmann, C. Loppacher, and H.-J. Güntherodt, J. Vac. Sci. Technol. B 14, 1280 (1996).

${ }^{17}$ O. Dudko, A. Filippov, J. Klafter, and M. Urbakh, Proc. Natl. Acad. Sci. U.S.A. 100, 11378 (2003).

${ }^{18}$ S. Krylov, K. Jinesh, H. Valk, M. Dienwiebel, and J. Frenken, Phys. Rev. E 71, 065101 (2005).

${ }^{19}$ S. Morita, S. Fujisawa, and Y. Sugawara, Surf. Sci. Rep. 23, 1 (1996).

${ }^{20}$ H. Hölscher, U. Schwarz, and R. Wiesendanger, Europhys. Lett. 36, 19 (1996). 